\title{
RELAÇÃO FAUNA DO SOLO-PAISAGEM EM PLANTIO DE EUCALIPTO EM TOPOSSEQUÊNCIA
}

\author{
Thaís de Andrade Corrêa Neto ${ }^{1}$, Lucia Helena Cunha dos Anjos ${ }^{1}$, Rodrigo Camara ${ }^{1}$, Marcos Gervasio Pereira ${ }^{1 *}$, \\ Maria Elizabeth Fernandes Correia ${ }^{2}$, Carlos Fellipe Siqueira Jaccoud ${ }^{1}$
${ }^{1}$ Universidade Federal Rural do Rio de Janeiro, Seropédica, Rio de Janeiro, Brasil - tacneto@ gmail.com; rcamara73@ gmail.com; lanjosrural@gmail.com; gervasio@ufrrj.br*; cjaccoud@hotmail.com
${ }^{2}$ Embrapa Agrobiologia, Seropédica, Rio de Janeiro, Brasil - elizabeth.correia@embrapa.br \\ Recebido para publicação: 02/09/2017 - Aceito para publicação 26/01/2018
}

\begin{abstract}
Resumo
Este estudo objetivou avaliar a influência do relevo sobre os artrópodes edáficos em um plantio de Eucalyptus urophylla. Coletou-se a camada de serapilheira e solo $(0-5 \mathrm{~cm})$ circunscritos a um gabarito metálico $(25 \times 25 \mathrm{~cm})$ lançado aleatoriamente $(\mathrm{N}=10)$ em três seções topográficas (TS, TM e TI: terços superior, médio e inferior, respectivamente) nas estações chuvosa e seca de 2003. Formicidae foi o grupo taxonômico predominante, independentemente da seção topográfica. A análise de componentes principais individualizou as seções topográficas entre si e demonstrou a correlação de um maior número de grupos taxonômicos com TS. A análise de agrupamento hierárquico indicou a elevada similaridade entre TI e TM, que se apresentaram como dissimilares em relação a TS. As diferenças entre as seções topográficas provavelmente foram influenciadas pela maior umidade gravimétrica e fertilidade do solo em TS.

Palavras-chave: Artrópodes do solo, biologia do solo, Eucalyptus urophylla, Formicidae, relevo.
\end{abstract}

\begin{abstract}
Soil fauna-landscape relationship in topossequence eucalyptus plantation. This study aimed to evaluate the influence of relief on edaphic arthropods in a Eucalyptus urophylla plantation. Litter layer and soil $(0-5 \mathrm{~cm})$ randomly released $(\mathrm{N}=10)$ circumscribed to a metal jig $(25 \times 25 \mathrm{~cm})$ were collected in three topographic sections (TS, TM, and TI: upper, middle, and lower thirds, respectively) in the rainy and dry seasons of 2003. Formicidae was the predominant taxonomic group, independently of the topographic section. The main component analysis individualized the topographic sections among themselves and demonstrated the correlation of a greater number of taxonomic groups with TS. The hierarchical clustering analysis indicated high similarity between TI and TM, which were dissimilar in relation to TS. The differences among topographic sections were probably influenced by the higher gravimetric moisture and soil fertility in TS.
\end{abstract}

Keywords. Soil arthropods, soil biology, Eucalyptus urophylla, Formicidae, relief.

\section{INTRODUÇÃO}

O relevo, ou posição topográfica, é capaz de alterar o conteúdo de água do solo em função da altitude e exposição ao sol, consequentemente modificando a temperatura do solo, bem como é responsável por variações no nível do lençol freático e intensidade dos processos de remoção e deposição de sedimentos (CHAGAS et al., 2013). Consequentemente, ocorrem alterações no padrão espacial das propriedades físicas e químicas do solo, dependendo da posição no relevo (MEIRELES et al., 2012). No terço inferior de encostas, por exemplo, a influência do nível do lençol freático é maior do que nos terços médio e superior, independentemente da composição textural. No entanto, a influência da textura também é um fator que condiciona a capacidade de retenção de água do solo, que é menor em camadas com textura arenosa, principalmente quando associada com baixo conteúdo de carbono (ANDRADE; STONE, 2011).

As flutuações no conteúdo de água e temperatura do solo são maiores nas camadas mais superficiais, onde se localiza a maior parte da fauna do solo. Os organismos que compõem essa comunidade atuam de maneira direta e indireta na ciclagem de nutrientes, por meio da regulação das populações de microrganismos decompositores e fragmentação da serapilheira, respectivamente (CORREIA; ANDRADE, 2008). Desta maneira, em sítios onde ocorre a exclusão experimental da fauna do solo, as taxas de decomposição da serapilheira são diminuídas, principalmente sob condições de déficit hídrico em ecossistemas florestais tropicais (CASTANHO et al., 2012). Esse resultado foi corroborado por outro estudo, segundo o qual as taxas de decomposição da serapilheira no inverno, período em que ocorre déficit hídrico, foram significativamente maiores no terço inferior, onde o conteúdo de água no

FLORESTA, Curitiba, PR, v. 48, n. 2, p. 213-224, abr/jun 2018

Neto. T. A. C. et.al.

ISSN eletrônico 1982-4688

DOI: $10.5380 /$ rf.v48 i2.55041 
solo foi maior em função da drenagem imperfeita, quando comparado aos terços superior e médio, que apresentam drenagem boa e moderada, respectivamente (CORRÊA NETO et al., 2014).

Dessa maneira, o relevo promove alterações na comunidade da fauna do solo e, consequentemente, pode interferir na dinâmica da ciclagem de nutrientes. Contudo, informações sobre a influência do relevo nos organismos do solo foram encontradas apenas para formigas da serapilheira em Floresta Amazônica de terra firme (VASCONCELOS et al., 2003). Estudos desta natureza são importantes, pois a fauna do solo fornece indicativos da sustentabilidade ecossistêmica uma vez que modificações edafoclimáticas resultam em alterações na estrutura e composição da comunidade da fauna do solo. Esse padrão foi verificado em estudos que englobam ecossistemas florestais com diferentes tamanhos (CAMARA et al., 2017), estágios de regeneração natural (MORAIS et al., 2010; CAMARA et al., 2012) e tipos de cobertura vegetal (MOÇO et al., 2005; CUNHA NETO et al., 2012; SILVA et al., 2013a). Também, em estudos sobre comparação entre áreas com diferentes tipos de manejo do solo (BARETTA et al., 2014) e manejo de plantas daninhas em Eucalyptus (GARLET et al., 2017), diferentes estações climáticas (MOÇO et al., 2005; CAMARA et al., 2012; SILVA et al., 2013b; BARETTA et al., 2014) e condições variadas de fertilidade do solo (BARETTA et al., 2014).

Este estudo objetivou avaliar a influência do relevo na comunidade de artrópodes edáficos em áreas de plantio de eucalipto. Testou-se a hipótese de que a comunidade de artrópodes do solo varia entre os terços da encosta em função do conteúdo de água no solo.

\section{MATERIAL E MÉTODOS}

O estudo foi conduzido em Seropédica, estado do Rio de Janeiro. O clima, tipo Aw (ALVARES et al., 2013), apresenta verão úmido e inverno seco, sendo a temperatura média anual de $24,6^{\circ} \mathrm{C}$ e a precipitação média anual de $1.200 \mathrm{~mm}$. Julho e agosto são os meses mais secos (PEREIRA et al., 2013). A vegetação primária é a Floresta Tropical Subcaducifólia (CORRÊA NETO et al., 2014).

Para o estudo, foi selecionado um plantio de Eucalyptus urophylla, instalado pela empresa Saint-Gobain, entre dezembro de 2001 e janeiro de 2002, com o objetivo de obter matéria-prima para a produção de carvão (CORRÊA NETO et al., 2007). Na implantação do plantio (espaçamento de 5 x $2 \mathrm{~m}$ ), foram realizadas as seguintes práticas: subsolagem; combate à matocompetição, com aplicação do herbicida glifosato; combate às formigas; e adubação mineral de pré-plantio com $240 \mathrm{~kg} \mathrm{ha}^{-1}$ da fórmula NPK (5-30-15) (CORRÊA NETO et al., 2007). Em 2002, foram realizadas gradagem e roçada mecânica nas áreas planas, roçada manual nas áreas de relevo ondulado, combate às formigas e adubação mineral de cobertura com $100 \mathrm{~kg} \mathrm{ha}^{-1}$ da fórmula NPK (20-00-20) para a manutenção do plantio.

Nesse plantio, instalado em uma área de relevo ondulado a suave-ondulado, foi selecionada uma topossequência composta por três seções: terço superior (TS), terço médio (TM) e terço inferior (TI). Em cada uma dessas seções, havia 250 covas. Os solos foram classificados em Argissolo Vermelho Amarelo (TS), Argissolo Amarelo (TM) e Planossolo Háplico (TI), respectivamente (CORRÊA NETO et al., 2014). O solo superficial $(0-20 \mathrm{~cm})$ entre as linhas de plantio em TS apresentou valores significativamente maiores de $\mathrm{pH}, \mathrm{Ca}^{+2}, \mathrm{Mg}^{+2}, \mathrm{Na}^{+}, \mathrm{K}^{+}$e $\mathrm{P}$, e valores menores de $\mathrm{Al}^{+3}$ e $\mathrm{H}+\mathrm{Al}$, com relação a TI e/ou TM (CORRÊA NETO et al., 2007).

Para a amostragem dos artrópodes edáficos, um gabarito metálico $(25 \times 25 \mathrm{~cm})$ foi lançado aleatoriamente 10 vezes (repetições) em cada seção. O material contido nessa seção $\left(625 \mathrm{~cm}^{2}\right)$ foi individualizado em serapilheira (camada de material orgânico disposto na superfície do solo) e solo (camada de $0-5 \mathrm{~cm}$ ), acondicionado em sacos plásticos previamente identificados e conduzidos ao laboratório para a extração dos organismos em extratores do tipo Berlese-Tüllgren ligeiramente modificados, conforme Souza et al. (2008). As amostras, coletadas nas estações chuvosa (verão) e seca (inverno) em 2003, permaneceram nos extratores por 10 dias consecutivos.

Os indivíduos do solo foram identificados em grandes grupos taxonômicos (classe, ordem, família) e quantificados. Para a análise da estrutura da comunidade da fauna do solo, foram estimadas as seguintes variáveis: densidade (indivíduos $\mathrm{m}^{-2}$ ) total e de grupos taxonômicos; riqueza (número de grupos) média e total; uniformidade (índice de Pielou); e diversidade (índice de Shannon). Formicidae foi considerado um grupo à parte de Hymenoptera, devido à facilidade de sua identificação. Os indivíduos adultos constituíram um grupo taxonômico separado de sua forma larval em função das diferenças entre esses estágios com relação ao papel ecológico desempenhado por eles no solo.

Avaliou-se a distribuição vertical dos organismos edáficos, que se refere ao adensamento dos animais nos compartimentos serapilheira ou solo em termos percentuais. Os grupos taxonômicos que apresentaram contribuição percentual inferior a $5 \%$ do total da comunidade de artrópodes em todas as seções topográficas e estações climáticas foram reunidos sob a denominação "Outros".

Com a finalidade de investigar possíveis influências das condições microclimáticas do solo sobre a fauna edáfica, as coletas desses organismos foram acompanhadas de avaliações de temperatura e conteúdo de água do solo. 
Para a estimativa do conteúdo de água, realizada pelo método da umidade gravimétrica (DONAGEMA et al., 2011), coletaram-se três réplicas de amostras de solo $(0-5 \mathrm{~cm})$ em cada seção, que foram secas em estufa $\left(105^{\circ} \mathrm{C}\right)$ em ambas as estações climáticas. A temperatura do solo também foi registrada no campo, com termômetro geotérmico digital, em três pontos de amostragem da fauna do solo por estação climática.

Os efeitos da seção topográfica (TI, TM, TS) e do compartimento (serapilheira, solo) sobre a comunidade de artrópodes edáficos foram avaliados dentro de cada estação climática (chuvosa, seca). Os valores de densidade total, densidade de grupos taxonômicos, riqueza média, umidade gravimétrica e temperatura do solo foram submetidos ao teste de Levene para verificar a homogeneidade das variâncias, e ao teste LSD para comparar as médias entre si. Quando a premissa de homogeneidade das variâncias não foi atendida, as médias foram comparadas entre si pelo teste não-paramétrico de Mann-Whitney (comparação entre duas médias) ou Kruskal-Wallis (comparação entre mais de duas médias). Essas análises estatísticas univariadas foram realizadas com o auxílio do programa STATISTICA versão 8.0.

Efetuou-se a análise multivariada de agrupamento hierárquico com o objetivo de identificar possíveis dissimilaridades entre as seções topográficas (TI, TM, TS), tendo como base seu efeito sobre a comunidade de artrópodes edáficos. Para tanto, empregou-se o método da distância de ligação de Ward e consideraram-se os valores dos atributos estruturais da comunidade (densidade total, riqueza média, riqueza total, uniformidade e diversidade), que foram obtidos com base na média entre os compartimentos (serapilheira, solo), além dos valores médios de umidade gravimétrica e temperatura do solo, em cada estação climática (chuvosa, seca).

Foram realizadas análises multivariadas de componentes principais para identificar possíveis correlações entre: (1) as seções topográficas (TI, TM, TS), os atributos estruturais da comunidade (média da densidade total, riqueza média, riqueza total, uniformidade e diversidade, entre os compartimentos serapilheira e solo), além dos valores médios de umidade gravimétrica e temperatura do solo, em cada estação climática (chuvosa, seca); (2) as seções topográficas (TI, TM, TS) e a abundância dos grupos taxonômicos (média entre os compartimentos serapilheira e solo) para cada estação climática (chuvosa, seca). As análises estatísticas multivariadas foram efetuadas com o auxílio do programa PAST versão 2.17 c.

\section{RESULTADOS}

Foram encontrados 24 grupos taxonômicos. Entre estes, 12 grupos predominaram, representando mais de 90\% de todos os artrópodes capturados: Formicidae, adultos de Coleoptera, larvas de Coleoptera, Diptera, Thysanoptera, Isopoda, Isoptera, Hymenoptera, Hemiptera, Araneae, Collembola, e Homoptera. Formicidae apresentou a maior contribuição percentual na comunidade em TM-SE (21\% e 34\%, nas estações chuvosa e seca, respectivamente), TS-SE (25\%, seca), TI-SO (49\% e 74\%, chuvosa e seca, respectivamente), TM-SO (36\% e 49\%, chuvosa e seca, respectivamente) e TS-SO (67\% e 52\%, chuvosa e seca, respectivamente).

Contudo, Coleoptera e Diptera apresentaram as maiores contribuições percentuais na comunidade em TISE, nas estações chuvosa (36\%) e seca (29\%), respectivamente. Essa posição foi ocupada por Hymenoptera em TS-SE, na estação chuvosa. O grupo Outros englobou os grupos taxonômicos Blattodea, Chilopoda, Dermaptera, Diplopoda, Diplura, larvas de Diptera, larvas de Lepidoptera, Neuroptera, Pseudoscorpionida, Symphyla, Thysanura, e Trichoptera.

A seção topográfica influenciou a densidade da maioria dos grupos predominantes. No entanto, houve variação nos resultados. O padrão de maior densidade em TM, em relação a TS, ocorreu para Formicidae (serapilheira, estação chuvosa), Coleoptera (solo, estações chuvosa e seca) e Isopoda (solo, estação seca) (Tabela 1). Maiores valores de densidade em TM, comparado com TI, foram verificados para Formicidae (serapilheira, estação chuvosa), Isopoda (serapilheira e solo, estação seca) e Araneae (serapilheira, estação seca).

Tabela 1. Densidade (indivíduos $\mathrm{m}^{-2} \pm$ erro padrão) de artrópodes na serapilheira e solo $(0-5 \mathrm{~cm})$ em diferentes seções no relevo (TI, TM e TS: terços inferior, médio e superior, respectivamente) em um plantio de Eucalyptus urophylla, em Seropédica (RJ), nas estações chuvosa e seca*.

Table 1. Arthropods density (individuals $\mathrm{m}^{-2} \pm$ standard error) for litter and soil $(0-5 \mathrm{~cm})$ in different relief sections (TI, TM, and TS: lower, middle, and upper thirds, respectively) of a Eucalyptus urophylla plantation, in the municipality of Seropédica, state of Rio de Janeiro, Brazil, in wet and dry seasons*.

\begin{tabular}{|c|c|c|c|c|c|c|}
\hline \multirow[t]{3}{*}{ Grupo taxonômico } & TI & TM & TS & TI & TM & TS \\
\hline & \multicolumn{3}{|c|}{ Serapilheira } & \multicolumn{3}{|c|}{ Solo } \\
\hline & \multicolumn{6}{|c|}{ Estação chuvosa } \\
\hline Formicidae & $16 \pm 1 \mathrm{Bb}$ & $45 \pm 1 \mathrm{~A}$ & $11 \pm 0 \mathrm{Bb}$ & $166 \pm 3 \mathrm{a}$ & $88 \pm 2$ & $224 \pm 7 a$ \\
\hline Coleoptera & $109 \pm 6$ & $43 \pm 1$ & $11 \pm 0$ & $19 \pm 0 \mathrm{AB}$ & $34 \pm 0 \mathrm{~A}$ & $11 \pm 0 \mathrm{~B}$ \\
\hline
\end{tabular}

FLORESTA, Curitiba, PR, v. 48, n. 2, p. 213-224, abr/jun 2018

Neto. T. A. C. et.al.

ISSN eletrônico 1982-4688

DOI: $10.5380 /$ rf.v48 i 2.55041 


\begin{tabular}{|c|c|c|c|c|c|c|}
\hline Diptera & $53 \pm 3$ & $19 \pm 1$ & - & $3 \pm 0$ & $11 \pm 0$ & $10 \pm 0$ \\
\hline Thysanoptera & $22 \pm 1$ & $29 \pm 2$ & $2 \pm 0$ & $8 \pm 0 \mathrm{~A}$ & $2 \pm 0 \mathrm{~B}$ & - \\
\hline Isopoda & $5 \pm 0$ & $8 \pm 0$ & $2 \pm 0$ & $35 \pm 1 \mathrm{~A}$ & $69 \pm 2 \mathrm{~A}$ & $3 \pm 0 \mathrm{~B}$ \\
\hline Isoptera & $3 \pm 0$ & - & $3 \pm 0$ & $2 \pm 0$ & - & $3 \pm 0$ \\
\hline Hymenoptera & $48 \pm 3$ & $35 \pm 1$ & $59 \pm 3$ & $42 \pm 1$ & $19 \pm 0$ & $11 \pm 0$ \\
\hline Larvas de Coleoptera & $5 \pm 0$ & - & $8 \pm 1$ & $3 \pm 0 \mathrm{~B}$ & - & $14 \pm 0 \mathrm{~A}$ \\
\hline Hemiptera & $16 \pm 0$ & $21 \pm 1$ & $6 \pm 0$ & $3 \pm 0$ & $10 \pm 0$ & $5 \pm 0$ \\
\hline Araneae & $10 \pm 0$ & - & $3 \pm 0$ & $3 \pm 0$ & $2 \pm 0$ & $5 \pm 0$ \\
\hline Collembola & $13 \pm 0$ & $6 \pm 0$ & $22 \pm 0$ & $35 \pm 2$ & $3 \pm 0$ & $10 \pm 0$ \\
\hline Homoptera & $2 \pm 0$ & $2 \pm 0$ & $2 \pm 0$ & - & - & $13 \pm 1$ \\
\hline \multirow[t]{2}{*}{ Outros } & $3 \pm 0$ & $6 \pm 0$ & $3 \pm 0$ & $18 \pm 1$ & $6 \pm 0$ & $34 \pm 1$ \\
\hline & \multicolumn{6}{|c|}{ Estação seca } \\
\hline Formicidae & $35 \pm 2 b$ & $91 \pm 5$ & $21 \pm 0$ & $424 \pm 10 \mathrm{a}$ & $189 \pm 8$ & $90 \pm 2$ \\
\hline Coleoptera & $10 \pm 0$ & $6 \pm 0$ & $6 \pm 0$ & $18 \pm 0 \mathrm{~A}$ & $25 \pm 1 \mathrm{~A}$ & $3 \pm 0 \mathrm{~B}$ \\
\hline Diptera & $46 \pm 1$ & $19 \pm 0$ & $11 \pm 0$ & $61 \pm 1 \mathrm{~A}$ & $5 \pm 0 \mathrm{~B}$ & $6 \pm 0 \mathrm{~B}$ \\
\hline Thysanoptera & $21 \pm 1$ & $64 \pm 2$ & $6 \pm 0$ & $5 \pm 0$ & $6 \pm 0$ & - \\
\hline Isopoda & $13 \pm 0 \mathrm{~B}$ & $29 \pm 1 \mathrm{~A}$ & - & $6 \pm 0 \mathrm{C}$ & $106 \pm 2 \mathrm{~A}$ & $24 \pm 1 B$ \\
\hline Isoptera & $26 \pm 1$ & $2 \pm 0$ & - & $10 \pm 0$ & - & $2 \pm 0$ \\
\hline Hymenoptera & - & $11 \pm 0$ & $10 \pm 0$ & $2 \pm 0$ & $21 \pm 1$ & $10 \pm 0$ \\
\hline Larvas de Coleoptera & $3 \pm 0 b$ & $21 \pm 1$ & $5 \pm 0$ & $21 \pm 0 \mathrm{a}$ & $16 \pm 0$ & $18 \pm 1$ \\
\hline Hemiptera & - & $3 \pm 0$ & - & $2 \pm 0$ & $2 \pm 0$ & - \\
\hline Araneae & $2 \pm 0 \mathrm{~B}$ & $11 \pm 0 \mathrm{~A}$ & $16 \pm 0 \mathrm{~A}$ & $13 \pm 1$ & $13 \pm 0$ & $13 \pm 0$ \\
\hline Collembola & - & - & $2 \pm 0$ & - & - & - \\
\hline Homoptera & $3 \pm 0$ & $5 \pm 0$ & $5 \pm 0$ & - & $2 \pm 0$ & - \\
\hline Outros & $2 \pm 0$ & $3 \pm 0$ & $3 \pm 0$ & $11 \pm 1$ & $5 \pm 0$ & $6 \pm 1$ \\
\hline
\end{tabular}

*Valores obtidos a partir da média de 10 réplicas. Médias seguidas de letras maiúsculas diferentes, dentro do compartimento e estação climática, indicam diferença significativa entre seções diferentes no relevo pelo teste LSD ou teste de Kruskal-Wallis ( $\mathrm{p}<0,05)$. Médias seguidas de letras minúsculas diferentes, dentro da seção no relevo e estação climática, indicam diferença significativa entre compartimentos pelo teste LSD ou de Mann-Whitney $(\mathrm{p}<0,05)$.

Em TI, a densidade foi maior para Thysanoptera (solo, estação chuvosa) e Diptera (solo, estação seca), quando comparado com TM (Tabela 1). Já a densidade de Araneae (serapilheira, estação seca) e de larvas de Coleoptera (solo, estação chuvosa) foi maior em TS, comparado com TI. Não houve efeito significativo da seção no relevo sobre a densidade de Collembola, Hemiptera, Homoptera, Hymenoptera, Isoptera, e Outros.

Verificou-se efeito significativo do compartimento apenas sobre a densidade de Formicidae e larvas de Coleoptera. Para o primeiro grupo taxonômico, a densidade no solo foi significativamente maior na comparação com a serapilheira (em TI, estações chuvosa e seca, e TS, estação chuvosa) (Tabela 1). A densidade de larvas de Coleoptera também foi maior no solo, com relação à serapilheira (em TI, estação seca).

A densidade total da comunidade foi significativamente maior em TM, comparado com TS, no compartimento serapilheira, estação chuvosa (Tabela 2). A riqueza média foi significativamente maior em TI, comparado com TS, na serapilheira, estação chuvosa. Houve tendência em maiores valores de riqueza total em TI (serapilheira, estação chuvosa; solo, estação seca), e menores valores em TM (serapilheira e solo, estação chuvosa). Em contrapartida, na serapilheira, estação seca, observou-se maior e menor valor de riqueza total em TM e TI, respectivamente.

Tabela 2. Densidade (indivíduos $\mathrm{m}^{-2} \pm$ erro padrão), riqueza média, riqueza total, uniformidade e diversidade de artrópodes na serapilheira e solo $(0-5 \mathrm{~cm})$ em diferentes seções no relevo (TI, TM e TS: terços inferior, médio e superior, respectivamente) em um plantio de Eucalyptus urophylla, em Seropédica (RJ), nas estações chuvosa e seca*.

Table 2. Density (individuals $\mathrm{m}^{-2} \pm$ standard error), average richness, total richness, uniformity, and arthropods diversity for litter and top soil $(0-5 \mathrm{~cm})$ in different relief sections (TI, TM, and TS: lower, middle, and upper thirds, respectively) of a Eucalyptus urophylla plantation, in the municipality of Seropédica, state of Rio de Janeiro, in wet and dry seasons*.

\begin{tabular}{lcccccc}
\hline & TI & TM & TS & TI & TM & TS \\
\cline { 2 - 7 } Atributos & \multicolumn{5}{c}{ Serapilheira } & \multicolumn{3}{c}{ Estação chuvosa } \\
\hline Densidade total & $304 \pm 12$ & $214 \pm 3$ & $133 \pm 3$ & $338 \pm 5$ & $243 \pm 2$ & $342 \pm 7$ \\
Riqueza média & $5 \mathrm{~A}$ & $4 \mathrm{AB}$ & $3 \mathrm{~B} \mathrm{~b}$ & 6 & 4 & $5 \mathrm{a}$ \\
Riqueza total & 14 & 11 & 13 & 14 & 11 & 17 \\
Uniformidade & 0,75 & 0,85 & 0,72 & 0,66 & 0,75 & 0,55 \\
Diversidade & 2,84 & 2,95 & 2,65 & 2,51 & 2,58 & 2,26 \\
\hline
\end{tabular}




\begin{tabular}{lcccccc}
\hline & \multicolumn{7}{c}{ Estação seca } \\
\hline Densidade total & $160 \pm 4 \mathrm{AB}$ & $266 \pm 6 \mathrm{~A}$ & $85 \pm 1 \mathrm{~B} \mathrm{~b}$ & $571 \pm 11$ & $386 \pm 10$ & $171 \pm 1 \mathrm{a}$ \\
Riqueza média & 3 & 4 & 4 & 5 & 5 & 3 \\
Riqueza total & 10 & 13 & 11 & 13 & 11 & 9 \\
Uniformidade & 0,81 & 0,75 & 0,85 & 0,30 & 0,68 & 0,79 \\
Diversidade & 2,70 & 2,76 & 2,94 & 1,11 & 2,37 & 2,52 \\
\hline
\end{tabular}

*Valores obtidos a partir da média de 10 réplicas. Médias seguidas de letras maiúsculas diferentes, dentro do compartimento e estação climática, indicam diferença significativa entre seções diferentes no relevo pelo teste LSD ou teste de Kruskal-Wallis ( $\mathrm{p}<0,05$ ). Médias seguidas de letras minúsculas diferentes, dentro da seção no relevo e estação climática, indicam diferença significativa entre compartimentos pelo teste LSD ou de Mann-Whitney $(\mathrm{p}<0,05)$

Verificou-se maior valor de riqueza total no solo em TS na estação chuvosa, enquanto que, na estação seca, ocorreu menor valor desse atributo (Tabela 2). A uniformidade tendeu a ser maior em TM e menor em TS na serapilheira e no solo, estação chuvosa. Já na estação seca, foram encontrados maiores valores de uniformidade em TS, na serapilheira e no solo, menores em TM, na serapilheira, e menores em TI, no solo. A diversidade foi maior em TM e menor em TS, na serapilheira e no solo, estação chuvosa. Em contrapartida, na estação seca, os maiores valores de diversidade ocorreram em TS e os menores em TI, tanto na serapilheira quanto no solo.

Tratando-se do efeito de compartimento, a densidade total e a riqueza média foram significativamente maiores no solo em relação à serapilheira em TS, estação seca e chuvosa, respectivamente (Tabela 2). Por um lado, a riqueza total tendeu a ser maior no solo na comparação com a serapilheira em TS, estação chuvosa, e em TI, estação seca. Por outro, na serapilheira, foram verificados maiores valores de riqueza total em TM e TS, estação seca (Tabela 2). A uniformidade e a diversidade foram maiores na serapilheira quando comparadas com as do solo, em todas as três seções topográficas, em ambas as estações climáticas.

A análise dos componentes principais para os atributos estruturais da comunidade indicou a individualização das seções topográficas entre si, por meio da relação entre o componente principal 1 (eixo 1) e o componente principal 2 (eixo 2), em ambas as estações climáticas (Figura 1). Na estação chuvosa, as seções TI e TM ficaram agrupadas na porção direita do eixo 1 (valores positivos), enquanto TS posicionou-se na porção esquerda do eixo 1 (valores negativos). Já na estação seca, as seções TI e TM agruparam-se na porção esquerda do eixo 1 (valores negativos), ao passo que TS posicionou-se na porção direita do eixo 1 (valores positivos). O eixo 1 foi responsável por explicar 58,06 \% e 64,97\% da variabilidade dos dados encontrados nas estações chuvosa e seca, respectivamente.

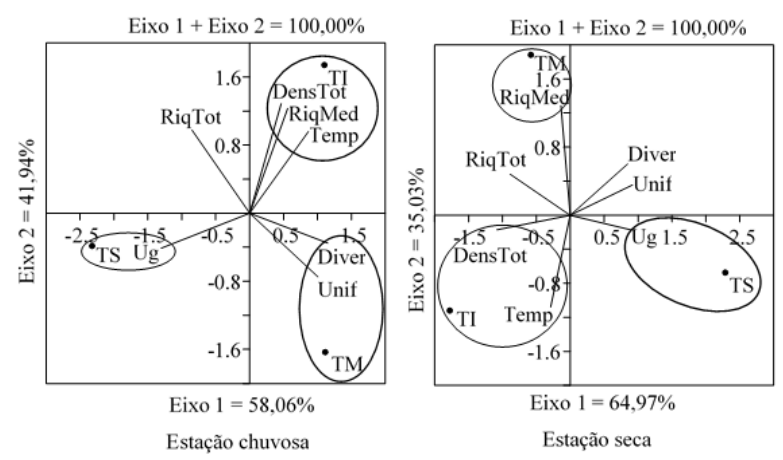

Figura 1. Análise dos componentes principais dos atributos estruturais da comunidade de artrópodes edáficos, temperatura e conteúdo de água do solo em diferentes seções no relevo (TI, TM e TS: terços inferior, médio e superior, respectivamente) em um plantio de Eucalyptus urophylla, em Seropédica (RJ), nas estações chuvosa e seca. DensTot: densidade total; Diver: diversidade, RiqMed: riqueza média; RiqTot: riqueza total; Unif: uniformidade; Temp: temperatura do solo; Ug: umidade gravimétrica (conteúdo de água do solo).

Figure 1. Main component analysis of the structural attributes of edaphic arthropod community, soil temperature and soil moisture in different relief sections (TI, TM, and TS: lower, middle, and upper thirds, respectively) of a Eucalyptus urophylla plantation, in the municipality of Seropédica, state of Rio de Janeiro, Brazil, in wet and dry season. DensTot: total density; Diver: diversity, RiqMed: average richness; RiqTot: total richness; Unif: uniformity; Temp: soil temperature; Ug: gravimetric moisture (soil water content).

FLORESTA, Curitiba, PR, v. 48, n. 2, p. 213-224, abr/jun 2018

Neto. T. A. C. et.al.

ISSN eletrônico 1982-4688

DOI: 10.5380/rf.v48 i2.55041 
O eixo 2 individualizou as seções topográficas TI e TM (Figura 1). Na estação chuvosa, a seção TI localizou-se na porção superior (valores positivos) e TM, na porção inferior (valores negativos). Na estação seca, houve inversão do posicionamento; TM posicionou-se na porção superior, ao passo que TI, na porção inferior. O eixo 2 explicou $41,94 \%$ e $35,03 \%$ da variabilidade dos dados nas estações chuvosa e seca, respectivamente. Em ambas as estações climáticas, o conjunto dos eixos 1 e 2 explicou $100 \%$ da variância observada.

A seção TI correlacionou-se com os atributos densidade total e temperatura do solo em ambas as estações climáticas e com a riqueza média na estação chuvosa (Figura 1). A seção TM apresentou correlação com a uniformidade e diversidade na estação chuvosa, e com a riqueza média na estação seca. Em contrapartida, TS correlacionou-se apenas com o conteúdo de umidade do solo em ambas as estações climáticas. O atributo riqueza total não se associou a nenhuma das seções topográficas.

A relação entre o componente principal 1 (eixo 1) e o componente principal 2 (eixo 2), na análise dos componentes principais para a densidade dos grupos taxonômicos, permitiu a separação entre as seções topográficas (Figura 2). Na estação chuvosa, as seções TI e TM ficaram agrupadas na porção esquerda do eixo 1 (valores negativos), enquanto TS posicionou-se na porção direita do eixo 1 (valores positivos). Contudo, o eixo 2 individualizou as seções topográficas TI e TM, uma vez que tais seções permaneceram na porção superior (valores positivos) e na porção inferior (valores negativos), respectivamente. Os eixos 1 e 2 explicaram 68,99\% e 31,02\% da variabilidade destes dados, respectivamente, e $100 \%$ da variabilidade, em conjunto.
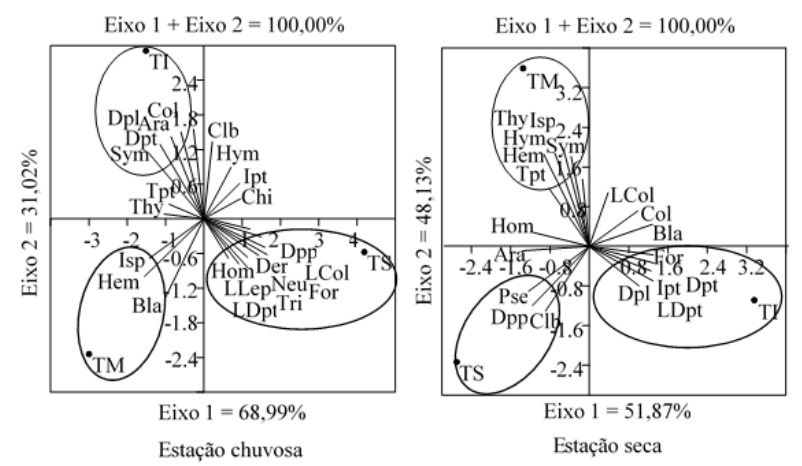

Figura 2. Análise dos componentes principais da densidade dos grupos taxonômicos de artrópodes edáficos em diferentes seções no relevo (TI, TM e TS: terços inferior, médio e superior, respectivamente) em um plantio de Eucalyptus urophylla, em Seropédica (RJ), nas estações chuvosa e seca. Ara: Araneae; Bla: Blattodea; Chi: Chilopoda; Col: Coleoptera; Clb: Collembola; Der: Dermaptera; Dpp: Diplopoda; Dpl: Diplura; Dpt: Diptera; For: Formicidae; Hem: Hemiptera; Hom: Homoptera; Hyme: Hymenoptera; Ipd: Isopoda; Ipt: Isoptera; LCol: larvas de Coleoptera; LDpt: larvas de Diptera; LLep: larvas de Lepidoptera; Neu: Neuroptera; Pse: Pseudoscorpionida; Sym: Symphyla; Tsp: Thysanoptera; Thy: Thysanura; Tri: Trichoptera.

Figure 2. Main component analysis for the density of taxonomic groups of edaphic arthropods in different relief sections (TI, TM, and TS: lower, middle, and upper thirds, respectively) in a Eucalyptus urophylla plantation, in the municipality of Seropédica, state of Rio de Janeiro, Brazil, in wet and dry seasons. Ara: Araneae; Bla: Blattodea; Chi: Chilopoda; Col: Coleoptera; Clb: Collembola; Der: Dermaptera; Dpp: Diplopoda; Dpl: Diplura; Dpt: Diptera; For: Formicidae; Hem: Hemiptera; Hom: Homoptera; Hyme: Hymenoptera; Ipd: Isopoda; Ipt: Isoptera; LCol: larvae of Coleoptera; LDpt: larvae of Diptera; LLep: larvae of Lepidoptera; Neu: Neuroptera; Pse: Pseudoscorpionida; Sym: Symphyla; Tsp: Thysanoptera; Thy: Thysanura; Tri: Trichoptera.

Entre os grupos taxonômicos, apenas três correlacionaram-se com TI (Araneae, Coleoptera e Diplura) e seis com TM (Blattodea, Hemiptera, Hymenoptera, Isopoda, Thysanoptera e Thysanura) (Figura 2). O maior número de grupos taxonômicos (nove) esteve correlacionado com TS, sendo eles: Collembola, Dermaptera, Diplopoda, Homoptera, larvas de Coleoptera, larvas de Lepidoptera, Neuroptera, Pseudoscorpionida, e Trichoptera.

O dendrograma de agrupamento hierárquico indicou a formação de dois macro grupos: um grupo formado pela reunião de TI (em ambas as estações climáticas) e TM (estação seca), e outro formado pela seção topográfica TS (em ambas as estações climáticas) e TM (estação chuvosa) (Figura 3). Esse resultado demonstrou a grande dissimilaridade entre TI e TS, que se configuraram como ambientes distintos entre si para os artrópodes edáficos. Por outro lado, a seção TS provavelmente apresentou condições intermediárias para esses organismos, na comparação com as demais seções topográficas. 


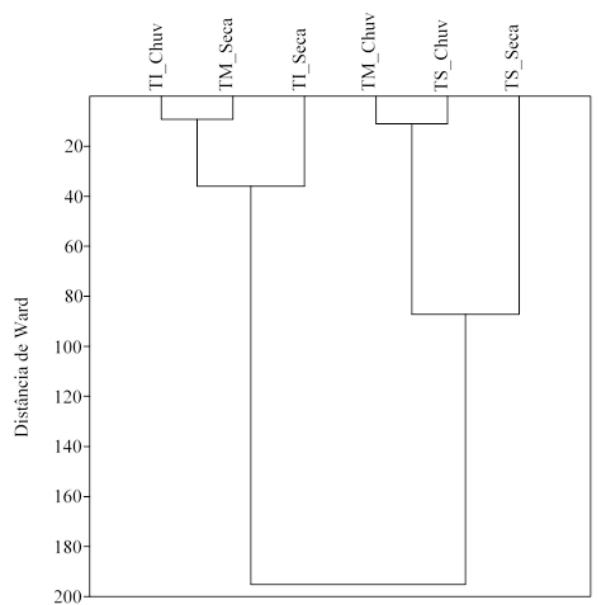

Figura 3. Dendrograma de agrupamento hierárquico para a comunidade de artrópodes edáficos em diferentes seções no relevo (TI, TM e TS: terços inferior, médio e superior, respectivamente) em um plantio de Eucalyptus urophylla, em Seropédica (RJ) nas estações chuvosa e seca.

Figure 3. Cluster dendrogram for the edaphic arthropod community in different relief sections (TI, TM, and TS: lower, middle, and upper thirds, respectively) of a Eucalyptus urophylla plantation, in the municipality of Seropédica, state of Rio de Janeiro, Brazil, in wet and dry seasons.

Em TI e TM, estação chuvosa, praticamente metade de todos os organismos foram capturados na serapilheira (Figura 4). No entanto, verificou-se que, em TS, estação chuvosa, e em todas as seções topográficas (TI, TM, TS), estação seca, a maior parte dos organismos foi capturada no solo, comparado com a serapilheira.
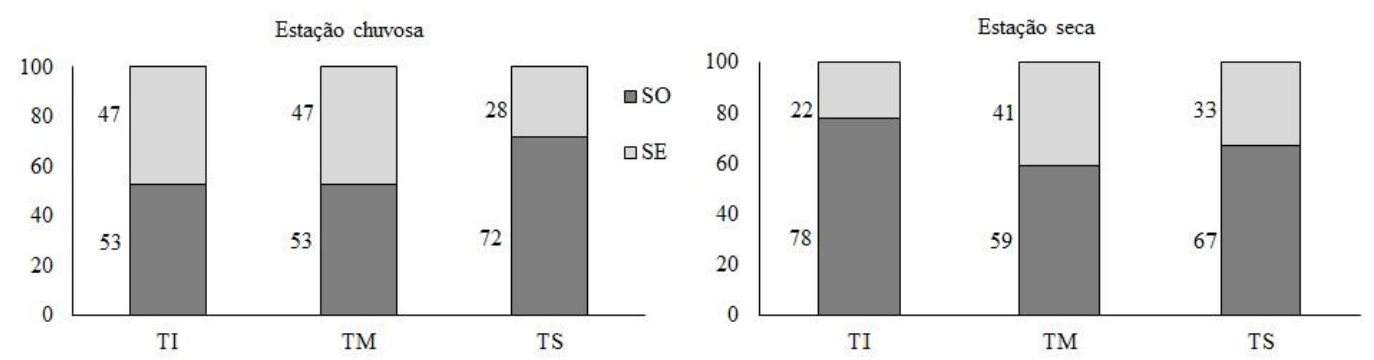

Figura 4. Distribuição percentual dos artrópodes do solo nos compartimentos serapilheira e solo $(0-5 \mathrm{~cm}), \mathrm{SE}$ e $\mathrm{SO}$, respectivamente, em diferentes seções no relevo (TI, TM e TS: terços inferior, médio e superior, respectivamente) em um plantio de Eucalyptus urophylla, em Seropédica, RJ, nas estações chuvosa e seca.

Figure 4. Percentage distribution of soil arthropods in litter and soil compartments $(0-5 \mathrm{~cm})$, SE and SO, respectively, in different relief sections (TI, TM, and TS: lower, middle, and upper thirds, respectively) of a Eucalyptus urophylla plantation, in the municipality of Seropédica, state of Rio de Janeiro, in wet and dry seasons.

Os valores médios de umidade gravimétrica do solo na estação chuvosa foram significativamente maiores em TS $(15,25 \%)$, em relação a TI $(4,73)$ e TM $(3,24)$, que não se diferenciaram entre si. Na estação seca, a umidade gravimétrica também foi significativamente maior em TS (16,78), quando comparado com TI (4,77\%). No entanto, não houve diferenças significativas entre esses valores e o verificado em TM $(8,90 \%)$, na referida estação climática.

Verificaram-se diferenças significativas entre as três seções topográficas com relação à temperatura média do solo, em ambas as estações climáticas. Na estação chuvosa, a temperatura do solo foi maior em TI $\left(30,2^{\circ} \mathrm{C}\right)$, menor em TS $\left(26,2^{\circ} \mathrm{C}\right)$ e apresentou valores intermediários em TM $\left(27,5^{\circ} \mathrm{C}\right)$. Na estação seca, embora a temperatura do solo também tenha sido maior em TI $\left(22,9^{\circ} \mathrm{C}\right)$, o menor valor foi observado em TM $\left(19,2^{\circ} \mathrm{C}\right)$ e o valor intermediário em TS $\left(22,1^{\circ} \mathrm{C}\right)$.

FLORESTA, Curitiba, PR, v. 48, n. 2, p. 213-224, abr/jun 2018

Neto. T. A. C. et.al.

ISSN eletrônico 1982-4688

DOI: $10.5380 /$ rf.v48 i2.55041 


\section{DISCUSSÃO}

Com exceção do compartimento serapilheira na seção topográfica TS, Formicidae foi o grupo predominante neste estudo. Isso pode indicar a necessidade de controle de formigas-cortadeiras no plantio, manejo que representa um considerável fator de custo na condução de plantios de eucalipto (GUEDES et al., 2011). O predomínio de formigas em regiões tropicais pode ser um reflexo da elevada riqueza de espécies (SILVA; SILVESTRE, 2004), aliado ao seu favorecimento ocasionado pela ampla variedade de recursos alimentares (detritívoras, predadoras, polinizadoras).

O predomínio de Formicidae também ocorreu em diferentes ecossistemas tropicais, nos quais se empregaram funis de Berlese-Tüllgren para a extração dos artrópodes da serapilheira e/ou do solo (MOÇO et al., 2005; MORAIS et al., 2010; SILVA et al., 2013a). Formicidae predominou: em um plantio de eucalipto Corymbia citriodora e em uma capoeira (regeneração natural de Mata Atlântica) no Parque Estadual do Desengano, na região norte-fluminense (MOÇO et al., 2005); em áreas de floresta secundária, floresta primária, sistema agroflorestal e roça na região da Amazônia brasileira (MORAIS et al., 2010); e em plantios puros de Eucalyptus camaldulensis e Acacia mangium instalados sobre cava de extração de argila em Campos dos Goytacazes, no estado do Rio de Janeiro (SILVA et al., 2013a).

Não foi observado um padrão claro do efeito da seção no relevo sobre a densidade dos grupos taxonômicos e atributos estruturais da comunidade de artrópodes edáficos (densidade total, riqueza média, riqueza total, uniformidade, diversidade). Tal resultado também ocorreu em uma área de Floresta Amazônica de Terra Firme, onde a riqueza de táxons de formigas numa área de vale (117 espécies) não apresentou diferenças estatísticas em relação a uma área de planalto (114 espécies). Esse padrão também foi observado para a diversidade (valores não informados) no estado do Amazonas (VASCONCELOS et al., 2003). De acordo com os autores citados, isso foi reflexo da influência de outros fatores, tais como a textura do solo, o estoque de serapilheira disposto sobre a superfície do solo, a densidade de árvores, a área basal, entre outros fatores, e é decorrente da complexidade das interrelações entre os atributos físicos, químicos e biológicos do solo.

As análises multivariadas empregadas indicaram diferenças entre as seções em ambas as estações climáticas. A análise multivariada de componentes principais evidenciou que tanto a seção TI quanto a seção TM correlacionaram-se com alguns atributos estruturais estudados. Dessa maneira, houve tendência de maiores valores dos referidos atributos em ambas as seções topográficas. Em contrapartida, TS não apresentou correlação com nenhum atributo da estrutura da comunidade.

$\mathrm{O}$ favorecimento da comunidade de artrópodes edáficos em TI foi, provavelmente, influenciado por maiores valores de temperatura do solo nessa seção topográfica. Esse resultado está em concordância com as taxas de decomposição da serapilheira, maiores em TI quando comparado com o observado em TS (CORRÊA NETO et al., 2014). Em ecossistemas florestais tropicais, o aumento da temperatura estimula a atividade da microbiota decompositora, o que concorre para acelerar as taxas de decomposição (DONNELLY et al., 1990).

Na última seção topográfica, ocorreu o aumento da densidade do maior número de grupos taxonômicos (nove), distribuídos em diferentes guildas tróficas: predadores (Dermaptera, Pseudoscorpionida), saprófagos (Diplopoda), saprófagos e/ou predadores (Neuroptera, larvas de Coleoptera, larvas de Lepidoptera), saprófagos e/ou micrófagos (Collembola), e herbívoros (Homoptera), além de organismos que não possuem funcionalidade conhecida no solo (Trichoptera). Isso foi corroborado pelo resultado da análise multivariada de agrupamento hierárquico, que indicou que há mais similaridades entre TI e TM do que entre essas seções topográficas e TS. Portanto, do ponto de vista da composição, a comunidade de artrópodes foi favorecida em TS.

Esse resultado foi, provavelmente, uma consequência dos maiores valores de umidade gravimétrica em TS, que refletem a maior capacidade de retenção de água no solo. Assim, a ocorrência de maiores valores de atributos da comunidade no compartimento solo foi comparativamente mais frequente em TS. Isso ocorreu devido aos maiores valores observados no solo dessa seção topográfica, comparado com a serapilheira, para os atributos densidade de Formicidae, riqueza média e riqueza total da comunidade na estação chuvosa, e densidade total de artrópodes na estação seca.

Em TS, a maior retenção de água na camada superficial do solo $(0-10 \mathrm{~cm})$ ocorreu em função de a textura ser mais argilosa nessa seção topográfica (25\% de argila, $60 \%$ de areia e $15 \%$ de silte, aproximadamente), enquanto que, em TI, a textura da camada superficial do solo é arenosa (8\% de argila, $85 \%$ de areia e $7 \%$ de silte, aproximadamente), conforme Balieiro et al. (2008). Esse resultado corroborou o estudo de Vasconcelos et al. (2003), conduzido em Floresta Amazônica brasileira, uma vez que a riqueza de espécies de formigas foi significativamente maior em áreas onde o solo apresentou textura argilosa, comparado com aquelas em que o solo é arenoso, provavelmente em função de diferenças na drenagem de água no perfil do solo.

Sob condições de maior conteúdo de água no solo, o crescimento de plantas e a produção de serapilheira foram favorecidos, o que concorre para o aumento na disponibilidade de recursos alimentares para os artrópodes do solo 
(MOÇO et al., 2005). Com isso, observou-se o padrão geral de elevado contingente de artrópodes encontrado na camada de serapilheira, comparado com a camada superficial de solo, durante a estação chuvosa. Já na estação seca, a maior parte dos organismos foi encontrada no solo. Acredita-se que a migração vertical descendente dos artrópodes na estação seca, isto é, da serapilheira para o solo, tenha ocorrido devido a condições microclimáticas mais favoráveis no solo, provavelmente em função de menores variações de umidade, quando comparado com a serapilheira. Esse comportamento dos artrópodes também foi verificado em ecossistemas florestais de restinga, associados ao bioma Floresta Atlântica, no estado do Rio de Janeiro (SILVA et al., 2013b).

Analogamente, tem-se verificado que a comunidade de artrópodes do solo apresenta maior complexidade durante a estação chuvosa, em relação à estação seca. Em dois plantios de eucalipto Corymbia citriodora abandonados e em diferentes estágio de regeneração natural de Mata Atlântica e em uma área de mata nativa, no estado do Rio de Janeiro, a abundância total, riqueza média e riqueza total foram maiores durante a estação chuvosa, comparado com a estação seca (CAMARA et al., 2012). No mesmo estado, a abundância de artrópodes edáficos também foi maior na estação chuvosa, em relação à estação seca, em plantios monoespecíficos de Eucalyptus grandis x Eucalyptus urophylla, Acacia mangium Wild e Mimosa artemisian (CUNHA NETO et al., 2012) e sob diferentes tipos de manejo do solo, em Santa Catarina (BARETTA et al., 2014).

A influência do compartimento também foi evidente com relação à uniformidade, cujos valores foram maiores (valores mais próximos de 1,0) na serapilheira em todas as seções topográficas, em ambas as estações climáticas. Isso indicou que não ocorreu a dominância marcada de um ou de poucos grupos em relação aos demais nesse compartimento. Consequentemente, a distribuição mais uniforme dos indivíduos nos grupos taxonômicos na serapilheira influenciou nos maiores valores de diversidade observados nesse compartimento, comparado com o solo. Acredita-se que esse padrão tenha sido decorrente da oferta de mais recursos alimentares na camada de serapilheira, com a consequente maior diversidade de artrópodes nesse compartimento. O oposto pode ter ocorrido no solo, onde os menores valores de uniformidade e diversidade ocorreram devido à predominância mais relevante de apenas um grupo (Formicidae).

Outro fator que pode ter contribuído para esse resultado são as melhores condições químicas do solo (maiores valores de $\left.\mathrm{pH}, \mathrm{P}, \mathrm{K}^{+}, \mathrm{Ca}^{+2}, \mathrm{Mg}^{+2}\right)$ na camada superficial $(0-20 \mathrm{~cm})$ em TS (CORRÊA NETO et al., 2007). Segundo esses autores, esse resultado é um reflexo da maior influência do material de origem, que fornece nutrientes com o intemperismo dos minerais primários em TS, enquanto as demais seções topográficas têm maior influência de sedimentos coluviais e aluviais já intemperizados. O favorecimento da comunidade de artrópodes em solos com maior fertilidade foi anteriormente observado por Baretta et al. (2014).

\section{CONCLUSÕES}

- Formicidae foi o grupo taxonômico predominante, independentemente da seção topográfica.

- A análise multivariada de componentes principais individualizou as seções topográficas entre si e demonstrou que um maior número de grupos taxonômicos esteve correlacionado com TS.

- A análise multivariada de agrupamento hierárquico demonstrou a elevada dissimilaridade de TS em relação a TI e TM, que obtiveram similaridade maior entre si.

- As diferenças entre as seções topográficas foram influenciadas, provavelmente, pela maior umidade gravimétrica e fertilidade do solo em TS.

\section{REFERÊNCIAS}

ALVARES, C. A.; STAPE, J. L.; SENTELHAS, P. C.; GONÇALVES, J. L. M.; SPAROVEK, G. Koppen's climate classification map for Brazil. Meteorologische Zeitschrift, Stuttgart, v. 22, n. 6, p. 711-728, 2013.

ANDRADE, R. S.; STONE, L. F. Estimativa da umidade na capacidade de campo em solos sob Cerrado. Revista Brasileira de Engenharia Agrícola e Ambiental, Campina Grande, v. 15, n. 2, p. 111-116, 2011.

BALIEIRO, F. C.; OLIVEIRA, W. C.; PEREIRA, M. G.; ANJOS, L. H. C.; PICCOLO, M. C.; JACCOUD, C. F. 2008. Fertilidade e carbono do solo e uso da água pelo eucalipto numa topossequência em Seropédica, RJ. Revista Árvore, Viçosa, v. 32, n. 1, p. 153-162, 2008.

BARETTA, D.; BARTZ, M. L. C., FACHINI, I.; ANSELMI, R.; ZORTÉA, T.; BARETTA, C. R. D. M. Soil fauna and its relation with environmental variables in soil management systems. Revista Ciência Agronômica, Fortaleza, v. 45, n. 5, p. 871-879, 2014.

FLORESTA, Curitiba, PR, v. 48, n. 2, p. 213-224, abr/jun 2018

Neto. T. A. C. et.al.

ISSN eletrônico 1982-4688

DOI: 10.5380/rf.v48 i2.55041 
CAMARA, R.; CORREIA, M. E. F.; VILLELA, D. M. Effects of eucalyptus plantations on soil arthropod communities in a Brazilian Atlantic Forest conservation. Bioscience Journal, Uberlândia, v. 28, n. 3, p. 445 - 455 , 2012.

CAMARA, R.; GOMES, J. M.; PEREIRA, M. G.; PIÑA-RODRIGUES, F. C. M. Atlantic forest size effect on structure and composition of soil arthropods community, RJ, Brazil. Floresta, Curitiba, v. 47, n. 2, p. 145-156, 2017.

CASTANHO, C. T.; LORENZO, L.; OLIVEIRA, A. A. The importance of mesofauna and decomposition environment on leaf decomposition in three forests in southeastern Brazil. Plant Ecology, Berlin, v. 213, n. 8, p. 1303-1313, 2012.

CHAGAS, C. S.; FONTANA, A.; CARVAlHO JUNIOR, W.; CAIRES, S. M. Atributos topográficos na diferenciação de Argissolos. Revista Brasileira de Ciência do Solo, Viçosa, v. 37, n. 6, p. 1441-1453, 2013.

CORRÊA NETO, T. A.; ANJOS, C. L. H.; PEREIRA, M. G.; AMORIM, H. B.; JACCOUD, C. F. S. Atributos edafoambientais e parâmetros dendrométricos de plantios de eucaplipto em uma topossequencia no campus da UFRRJ - Seropédica (RJ). Ciência Florestal, Santa Maria, v. 17, n. 1, p. 43-51, 2007.

CORRÊA NETO, T. A.; ANJOS, L. H. C.; PEREIRA, M. G. P.; JACCOUD, C. F. S. Aporte de serapilheira em plantios de eucalipto em função da qualidade do sítio. Pesquisa Florestal Brasileira, Colombo, v. 34, n. 80, p. 399-406, 2014

CORREIA, M. E. F.; ANDRADE, A. G. Formação de serapilheira e ciclagem de nutrientes. In: SANTOS, G. A.; CAMARGO, F. A. O. Fundamentos da matéria orgânica do solo: ecossistemas tropicais e subtropicais. Porto Alegre: Gênesis, p. 137-170, 2008.

CUNHA NETO, F. V.; CORREIA, M. E. F.; PEREIRA, G. H. A.; PEREIRA, M. G.; LELES, P. S. S. Soil fauna as an indicator of soil quality in forest stands, pasture and secondary forest. Revista Brasileira de Ciência do Solo, Viçosa, v. 36, n. 5, p. 1407-1417, 2012.

DONAGEMA, G. K.; CAMPOS, D. V. B.; CALDERANO, S. B.; TEIXEIRA, W. G.; VIANA, J. H. M. Manual de métodos de análise de solo. Rio de Janeiro, Embrapa Solos, 2011. 225 p.

DONNELLY, P. K.; ENTRY, J. A.; CRAWFORD, D. L.; CROMACK JR, K. Cellulose and lignin degradation in forest soils: response to moisture, temperature and acidity. Microbial Ecolology, Berlin, v. 20, n. 1, p. 289-295, 1990.

GARLET, J.; COSTA, E. C.; BOSCARDIN, J.; MACHADO, D. N.; PEDRON, L.; MACHADO, L. M. Fauna edáfica em plantio inicial de Eucalyptus sob diferentes alternativas de controle de plantas daninhas. Pesquisa Florestal Brasileira, Colombo, v. 37, n. 91, p. 403-408, 2017.

GUEDES, I. C. L.; COELHO JÚNIOR, L. M.; OLIVEIRA, A. D.; MELLO, J. M.; REZENDE, J. L. P.; SILVA, C. P. C. Economic analysis of replacement regeneration and coppice regeneration in eucalyptus stands under risk conditions. Cerne, Lavras, v. 17, n. 3, p. 393-401, 2011.

MEIRELES, H. T.; MARQUES JÚNIOR, J.; CAMPOS, M. C. C.; PEREIRA, G. T. Relações solo-paisagem em topossequência de origem basáltica. Pesquisa Agropecuária Tropical, Goiânia, v. 42, n. 2, p. 129-136, 2012.

MOÇO, M. K.; GAMA-RODRIGUES, E. F.; GAMA-RODRIGUES, A. C.; CORREIA, M. E. F. Caracterização da fauna edáfica em diferentes coberturas vegetais na região Norte Fluminense. Revista Brasileira de Ciência do Solo, Viçosa, v. 29, n. 4, p. 555-564, 2005.

MORAIS, J. W.; OLIVEIRA, V. S.; DAMBROS, C. S.; TAPIA-CORAL, S. C.; ACIOLI, A. N. S. Mesofauna do solo em diferentes sistemas de uso da terra no Alto Rio Solimões, AM. Neotropical Entomology, Londrina, v. 39, n. 2, p. 145-152, 2010.

PEREIRA, W.; LEITE, J. M.; HIPÓLITO, G. S.; SANTOS, C. L. R.; REIS, V. M. Acúmulo de biomassa em variedades de cana-de-açúcar inoculadas com diferentes estirpes de bactérias diazotróficas. Revista Ciência Agronômica, Fortaleza, v. 44, n. 2, p. 363-370, 2013.

SILVA, R. R.; SILVESTRE, R. Riqueza da fauna de formigas (Hymenoptera: Formicidae) que habita as camadas superficiais do solo em Seara, Santa Catarina. Papéis Avulsos de Zoologia, São Paulo, v. 44, n. 1, p. 1-11, 2004. 
SILVA, C. F.; MARTINS, M. A.; SILVA, E. M. R.; PEREIRA, M. G.; CORREIA, M. E. F. Influência do sistema de plantio sobre atributos dendrométricos e fauna edáfica, em área degradada pela extração de argila. Revista Brasileira de Ciência do Solo, Viçosa, v. 37, n. 6, p. 1742-1751, 2013a.

SILVA, C. F.; PEREIRA, G. H. A.; PEREIRA, M. G.; SILVA, A. N. Fauna edáfica em área periodicamente inundável na restinga da Marambaia, RJ. Revista Brasileira de Ciência do Solo, Viçosa, v. 37, n. 3, p. 587-595, $2013 b$.

SOUZA, R. C.; CORREIA, M. E. F.; PEREIRA, M. G.; SILVA, E. M. R.; PAULA, R. R.; MENEZES, L. F. T. Estrutura da comunidade da fauna edáfica em fragmentos florestais na Restinga da Marambaia, RJ. Revista Brasileira de Ciências Agrárias, Recife, v. 3, n. 1, p. 49-57, 2008.

VASCONCELOS, H. L.; MACEDO, A. C. C.; VILHENA, J. M. S. Influence of topography on the distribution of ground-dwelling ants in an Amazonian Forest. Studies on Neotropical Fauna and Environment, London, v. 38, n. 2, p. 115-124, 2003. 
FLORESTA, Curitiba, PR, v. 48, n. 2, p 213-224, abr/jun 2018

Neto. T. A. C. et.al.

ISSN eletrônico 1982-4688 